\title{
Uso de la inmunohistoquímica para la búsqueda de proteínas priónicas en tercer párpado de ovinos en Chile ${ }^{\#}$
}

\author{
Use of immunohistochemestry to search prion protein in third eyelid of sheep in Chile
}

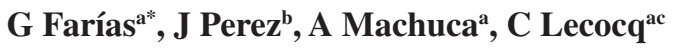 \\ aDepartamento de Patología Animal, Facultad de Ciencias Veterinarias y Pecuarias, Universidad de Chile, Santiago, Chile. \\ bDepartamento de Anatomía y Anatomía Patológica Comparadas, Facultad de Ciencias Veterinarias, \\ Universidad de Córdoba, Córdoba, España. \\ 'Servicio Agrícola y Ganadero, Unidad de Patología Animal del Departamento Laboratorios \\ y Estaciones Cuarentenarias Pecuarias, Santiago, Chile.
}

\begin{abstract}
SUMMARY
An immunohistochemstry (IHC) method to detect scrapie in sheep third eyelid lymphoid tissue was applied in post mortem animals. This method would allow to carry out the diagnosis in Chile, including the preclinical phase of the disease in sheep flock. Eighty samples from two year old sheep were obtained. Fourty two showed neurological signs consistent with scrapie and thirty eight came from slaughterhouse. Another thirteen blind samples were provided by a Reference Center (RC) in addition to sixteen obex used as controls. Samples were evaluated in sections with hematoxilin-eosin and classified as suitable if presenting at least four lymphoid follicles in the tissue. Based on this, seventy four out of ninety samples were considered suitable. The IHC results showed that all samples were negative to scrapie and only seven provided by the RC were positive. All obex samples were positive to immunostaining by $\mathrm{PrP}^{\mathrm{Sc}}$, unlike negative controls. The results demosntrate that this diagnostic tool may be considered for monitoring the sanitary condition related to scrapie in the ovine flock in Chile.
\end{abstract}

Key words: scrapie, prion, TSE, IHC.

\section{RESUMEN}

En este trabajo se implementó y aplicó un método para detectar el scrapie, utilizando la técnica de inmunohistoquímica (IHQ) en tejido linfoide de tercer párpado ovino. Este método permitiría realizar el diagnóstico en animales vivos, incluso en fases preclínicas de esta enfermedad en la masa ovina nacional. Se obtuvieron y procesaron 80 muestras de tercer párpado de ovinos nacionales mayores de 2 años, 42 con sintomatología nerviosa compatible con scrapie y 38 que fueron obtenidos de mataderos. Otras 13 muestras ciegas de validación de tercer párpado y sus respectivos óbex, además de 16 muestras de óbex utilizadas como controles, fueron aportadas por un Centro de Referencia (CR). Las muestras fueron evaluadas en cortes teñidos con Hematoxilina-Eosina y clasificadas como aptas cuando presentaban al menos 4 folículos linfoides en el tejido obtenido. Sobre esta base, 74 de las 93 muestras totales de tercer párpado fueron consideradas como aptas. Los resultados de IHQ mostraron que todas las muestras nacionales fueron negativas a scrapie y sólo 7 de las proporcionadas por el CR fueron positivas. Además, en todos los controles positivos de óbex se observó inmunomarcaje de $\mathrm{PrP}^{\mathrm{Sc}}$, lo cual no se evidenció en los controles negativos. Los resultados descritos anteriormente permiten demostrar la aplicabilidad y capacidad para utilizar esta herramienta diagnóstica en la masa ovina, lo cual permitiría establecer la condición del país respecto al scrapie y obtener los beneficios de esta situación sanitaria.

Palabras clave: scrapie, prion, EETs, IHQ.

\section{INTRODUCCIÓN}

El scrapie de los ovinos y caprinos es el prototipo de las encefalopatías espongiformes transmisibles (EETs), enfermedades neurodegenerativas fatales que afectan al

Aceptado: 18.04.2013.

\# Proyecto FIV 12101401.9102.009 Dirección de Investigación Facultad de Ciencias Veterinarias y Pecuarias, Universidad de Chile.

* Casilla 2, correo 15, Av. Santa Rosa 11735, La Granja, Santiago, Chile; gfarias@uchile.cl sistema nervioso central (SNC), que ha sido descrita en diferentes especies mamíferas incluida la humana. El agente etiológico es el prión $\left(\mathrm{PrP}^{\mathrm{Sc}}\right)$, isoforma alterada e infectiva de una proteína constitutiva de la membrana celular $\left(\mathrm{PrP}^{\mathrm{C}}\right)$ que presenta alta resistencia a métodos de inactivación, incapacidad de producir respuesta inmune en el organismo afectado y además convierte la isoforma celular en patológica (Aguzzi y col 2008, Farías y col 2009, Tranulis y col 2011).

La transmisión del scrapie ocurre principalmente por exposición oral y sus principales lesiones están restringidas al SNC. Su patogenia implica primero una fago- 
citosis por las células dendríticas foliculares a nivel intestinal, seguida de una replicación de los priones en los órganos linfoides, previo a la neuroinvasión (Jeffrey y González 2007, van Keulen y col 2008). Esto explica su largo periodo de incubación, posterior al cual recién es posible el diagnóstico con la aparición gradual de los signos clínicos, como cambio de comportamiento, prurito y alteraciones neurológicas. Sin embargo, la suceptibilidad y la patogenia en las ovejas se ven influenciadas por una predisposición genética, por los polimorfismos en los codones 136, 154 y 171 del gen PRNP de la proteína priónica, que determina mayor susceptibilidad a manifestar la enfermedad en los haplotipos VRQ y ARQ y menor para ARR (Fediaevsky y col 2008, Ortiz-Pelaez y Bianchini 2011).

Las EETs se diagnosticaban por las lesiones histopatológicas del SNC, las que incluyen cambios espongiformes, gliosis astrocítica y placas amiloides en el cerebro de animales y humanos (Jeffrey y González 2007, Vidal y col 2009). Actualmente la técnica gold standar para el diagnóstico es la inmunohistoquímica (IHQ) que se utiliza para detectar la presencia de la $\mathrm{PrP}^{\mathrm{Sc}}$ in situ en el cerebro. Además en algunas especies puede ser aplicada para analizar su distribución en los tejidos linfoides (Grassi y col 2008, Farías y col 2009, Monleón y col 2011).

La característica de la patogenia del scrapie clásico que involucra una acumulación temprana del prión en tejidos linfoides, antes de su propagación al SNC, representa la base para el diagnóstico preclínico mediante técnicas de IHQ. Éstas pueden ser realizadas en muestras in vivo o post mortem del tejido linfoide de tonsilas, linfonodos retrofaríngeos, tercer párpado o mucosa rectal (O' Rourke y col 2002, Monleón y col 2005, Vargas y col 2006, González y col 2008). Según O’ Rourke y col (2000, 2002) el tercer párpado sería más fácilmente accesible en animales vivos. En la actualidad, Chile cuenta con herramientas diagnósticas como son la histopatología tradicional, IHQ, ELISA y Western blot, entre otras. El gran inconveniente de estos métodos es que utilizan muestras de cerebro, por lo que deben ser realizados post mortem. Esto impide detectar la enfermedad en animales que pueden estar incubándola sin sintomatología clínica, lo que no permite un manejo adecuado (Grassi y col 2008, Farías y col 2009).

En contraste a la EET del bovino, el alto linfotropismo de los priones en el scrapie clásico, en la variante de la enfermedad Creutzfeldt-Jakob (vECJ) y en la enfermedad del desgaste crónico (Aguzzi y col 2008, Gilch y col 2011, Wadsworth y col 2011), ha permitido el uso de la IHQ para detectar la $\mathrm{PrP}^{\mathrm{Sc}}$ en tejido linfoide, antes de que se inicien las manifestaciones clínicas, permitiendo así realizar su diagnóstico preclínico (González y col 2008, Monleón y col 2011).

En base a estos antecedentes y las posibilidades futuras en el campo del diagnóstico, la prevención y el control de las EETs, se planteó el objetivo de este estudio de implementar y aplicar la detección de la proteína priónica en tejido linfoide de tercer párpado ovino mediante IHQ. Esta técnica poco invasiva, permitiría realizar un diagnóstico en animales vivos. Es importante destacar la necesidad de contar con herramientas de diagnóstico preclínico para estas enfermedades, puesto que en el país, la especie ovina se comercializa muchas veces antes de que exista cualquier posibilidad de que se manifieste la sintomatología del scrapie.

\section{MATERIAL Y MÉTODOS}

Se estudiaron un total de 93 muestras de tercer párpado de ovinos mayores de 2 años, obtenidas post mortem, sin importar raza o sexo. De éstas, 42 se obtuvieron en la unidad de patología del Servicio Agrícola y Ganadero, de animales con sintomatología compatible a scrapie, 38 fueron obtenidas en matadero y 13 muestras ciegas de validación de tercer párpado con sus 13 óbex respectivos, estas últimas aportadas por el centro de referencia (CR) canadiense (National Reference Laboratory for Scrapie and CWD, Animal Research Institute and Canadian Food Inspection Agency), además de los 16 controles de óbex de ovinos, 8 positivos y 8 negativos, provenientes del mismo centro.

De cada animal se obtuvo una biopsia de tejido linfoide de tercer párpado, según la técnica descrita por O'Rourke y col (2002), las muestras fueron fijadas en formalina neutra tamponada $(10 \%)$, se incluyeron en parafina y se procesaron en cortes seriados de $5 \mu \mathrm{m}$ de espesor, para el examen histológico y la posterior detección por inmunohistoquímica (IHQ) de la $\mathrm{PrP}^{\mathrm{Sc}}$.

\section{HISTOLOGÍA}

Previo a la realización de la IHQ, se procedió a evaluar la aptitud de las muestras, de acuerdo a la cantidad de folículos linfoides con centros germinales presentes en ellas. Las secciones de tejidos fueron teñidas con Hematoxilina y Eosina (H-E), siguiendo el procedimiento histológico estándar. Al examen microscópico (objetivo 10x) de cada muestra, se contó el número de folículos linfoides con centro germinal y la clasificación de su aptitud, se estableció de acuerdo a los criterios descritos por O’Rourke y col (2000).

\section{INMUNOHISTOQUÍMICA}

Como se utiliza un método de marcaje con peroxidasa, se inhibió la actividad endógena de esta enzima en el tejido, para ello las muestras se mantuvieron en una solución de agua oxigenada (Merck/Alemania) al 3\% en metanol absoluto (Merck/ Alemania) por $10 \mathrm{~min}$ y se lavaron en agua desionizada. Los siguientes tratamientos se realizaron para exponer los antígenos, descontaminar y aumentar la sensibilidad de la inmunotinción: 1) inmer- 
sión en ácido fórmico (Merck Alemania) al 98\% por 5 min, 2) lavado con una solución tampón de TBST pH 7,6 (Tris Buffer Salino Tween-20) 3 veces por 2 min, 3) incubación en una solución recuperadora de antígenos pH 6,1 (Target Retrieval Solution $\AA$ ) en autoclave por $20 \mathrm{~min}$ a $120^{\circ} \mathrm{C}$, una vez frías, se lavaron en una solución de TBST pH 7,6 por 10 min. La IHQ se realizó por capilaridad, los portaobjetos fueron pareados y montados en un sostenedor siguiendo los procedimientos indicados y con los reactivos suministrados por el kit Pullman TSE-IHC/99 (VRMD/USA) (VRMD 2004). Para la inmunotinción se incluyeron los siguientes pasos, cada uno separado por 3 lavados con tampón TBST pH 7,6: 1) tratamiento con una solución de proteinasa K (VRMD/USA) $(4 \mathrm{mg} / \mathrm{ml})$ por $90 \mathrm{seg}$; 2) incubación con el primer anticuerpo monoclonal (F99/97.6.1) (1:1000), durante $15 \mathrm{~min}$ a $37^{\circ} \mathrm{C}$ (Spaker y col 2002); 3) incubación con una solución del segundo anticuerpo policlonal anti-ratón biotinilado por 10 min; 4) y en streptavidina conjugada con peroxidasa HRP (Horseradish Peroxidase) por $10 \mathrm{~min}$. Para revelar y poner de manifiesto la inmunoreacción, se incubaron en oscuridad con el sustrato-cromógeno AEC (3-amino9-etilcarbazol) por $20 \mathrm{~min}$. La reacción se detuvo con lavados de agua bidestilada. Las muestras fueron teñidas con Hematoxilina de Mayer (Dako®) por 10 min. Todas las incubaciones se realizaron a temperatura ambiente, a menos que se indique lo contrario. Las muestras fueron procesadas de acuerdo a lo descrito en O'Rourke y col (2002) y las recomendaciones del kit Pullman TSEIHC/99 (VRMD 2004). Como controles positivos y negativos se utilizaron los cortes de óbex, aportados por el CR. Además se usaron como controles negativos cortes seriados en los que el primer anticuerpo fue sustituido por TBTS.

\section{ANÁLISIS DE LAS MUESTRAS}

Estudio histológico. Una vez realizada la tinción de H-E siguiendo las pautas de O'Rourke y col (2000), se estableció la aptitud de las muestras para realizar la IHQ. Apta: aquella en que a la observación microscópica se identificaran 4 o más folículos linfoides. No apta: cuando se identificaron menos de 4 folículos linfoides.

Estudio inmunohistoquímico. La tinción positiva del tejido linfoide de tercer párpado se identificó por una fuerte inmunotinción citoplasmática de patrón granular en los centros germinales de los folículos linfoides. Se consideraron positivas aquellas muestras en las que uno o más folículos mostraron el precipitado granular rojo de la inmunotinción. En las muestras consideradas negativas no se observó inmunotinción del tejido, en el mínimo de 4 centros germinales requeridos para el diagnóstico (O'Rourke y col 2002). Todas las muestras analizadas se clasificaron y fotografiaron, en un microscopio Axio Imagen A1 (Zeiss), con cámara fotográfica digital (Canon PowerShot G6).

\section{RESULTADOS Y DISCUSIÓN}

En este estudio se analizaron 93 muestras totales de tejido linfoide de tercer párpado (nacionales y CR), las cuales se observaron mediante la tinción de H-E, permitiendo clasificar 74/93 muestras aptas y 19/93 como no aptas, para posteriormente continuar con la IHQ. Esta clasificación se basó en el número de folículos linfoides con centros germinales presentes en las muestras según los criterios descritos por O'Rourke y col (2000). A pesar que se puede diagnosticar la enfermedad con un único folículo linfoide, en regiones con baja probabilidad de encontrar casos de scrapie, es necesaria la observación de al menos 4 folículos linfoides para diagnosticar correctamente una muestra como negativa. Si bien la $\mathrm{PrP}^{\mathrm{Sc}}$ se ubica en el centro germinal de los folículos linfoides, su presencia temprana en este tejido no es homogénea (van Keulen y col 2008, Monléon y col 2011). El examen histológico permitió establecer que no todas las muestras obtenidas presentaban el número mínimo de folículos linfoides, como se muestra en el cuadro 1.

$\mathrm{Al}$ analizar la clasificación de aptitud de las muestras, los resultados obtenidos muestran un 79,6\% de muestras aptas disponibles para la IHQ, lo que supone un porcentaje ligeramente inferior al $85 \%$ obtenido en el estudio de O'Rourke y col (2000). Estas diferencias se pueden atribuir a la dificultad en la obtención del tejido necesario en las primeras muestras, debido a inexperiencia y a la distribución irregular del tejido linfoide en tercer párpado.

Inmunohistoquímica: las 74 muestras aptas fueron analizadas mediante IHQ para detectar $\mathrm{PrP}^{\mathrm{Sc}}$ en el tejido linfoide de tercer párpado. Ninguna de las obtenidas en el país resultaron positivas (0/61) a la inmunoreacción con la proteína priónica patológica, al no observarse inmunomarcaje en ninguno de los folículos linfoides. Por otra parte, de las 13 muestras de tejido linfoide de tercer párpado aportadas por el CR, 7/13 fueron positivas en las que se visualizó el

Cuadro 1. Aptitud de muestras de tercer párpado de ovinos, según el número de folículos linfoides con centros germinales en cortes teñidos con H-E.

Aptitude of third eyelid samples of sheep according to number of lymphoid follicles with germinal centers in H-E stained sections.

\begin{tabular}{|c|c|c|c|c|c|c|c|}
\hline \multirow{2}{*}{$\begin{array}{l}\text { Origen de las } \\
\text { muestras }\end{array}$} & \multicolumn{4}{|c|}{ No aptas } & \multicolumn{2}{|c|}{ Aptas } & \multirow[t]{2}{*}{ Tota } \\
\hline & 0 & 1 & 2 & 3 & 4 & $>4$ & \\
\hline De matadero & 2 & 2 & 5 & 5 & 9 & 15 & 38 \\
\hline $\begin{array}{l}\text { Con signos } \\
\text { compatibles }\end{array}$ & 1 & 1 & 1 & 2 & 11 & 26 & 42 \\
\hline $\begin{array}{l}\text { Del centro de } \\
\text { referencia }\end{array}$ & 0 & 0 & 0 & 0 & 3 & 10 & 13 \\
\hline Total & & & & & & 74 & 93 \\
\hline
\end{tabular}


inmunoprecipitado de color rojo en los folículos linfoides, y 6/13 negativas, resultados que coincidieron en todos los casos con los de sus respectivos óbex. Además, todos los controles positivos de óbex de ovinos utilizados para IHQ presentaron inmunotinción, mientras que en los negativos nunca se detectó, según la pauta de O’Rourke y col (2002). Estos resultados, confirman la alta especificidad de casi $100 \%$ descrita para esta técnica en el diagnóstico de scrapie, utilizando tejido linfoide obtenido de tercer párpado, respecto a la técnica estándar de confirmación post mortem reconocidas por la OIE (Monléon y col 2005). Sin embargo, la sensibilidad descrita es muy diversa, entre un 44\% (Monléon y col 2011) hasta un 97,6\% (O’Rourke y col 2000), dependiendo del número y la edad de los ovinos utilizados. En el presente estudio descartamos algunos factores como el tamaño inadecuado de la muestra y la edad de los ovinos, que en este caso fue homogénea entre los 2 a los 4 años, por lo que los falsos negativos podrían deberse a la ausencia o escasa cantidad de $\mathrm{PrPsc}^{\mathrm{Sc}}$ en el tejido linfoide, a la distribución no homogénea de ésta en el sistema linforeticular (SLR) (van Keulen y col 2008), o a la presencia de otra cepa de prión, como la Nor98, que difiere del scrapie clásico en la presentación clínica y en el linfotropismo del prión, por lo que se postula que una vez que ingresa al digestivo invadiría directamente el SNC (Benestad y col 2008, Monléon y col 2011, Tranulis y col 2011).

En la figura 1, se presentan las microfotografías obtenidas del tejido linfoide de tercer párpado de las muestras estudiadas, donde se observa la inmunomarca en las muestras positivas (del CR) y la ausencia de ésta en las negativas (muestras nacionales). En la figura 2, se observan los cortes de la médula oblongada a nivel del óbex de ovinos utilizados como controles para la IHQ, uno positivo que muestra la inmunoreacción positiva de la proteína priónica patológica (inmunoprecipitado rojo), además de la visualización de numerosas vacuolas en el núcleo del tracto solitario, y otro negativo en que con la tinción de contraste es posible evidenciar los somas neuronales.

Los resultados obtenidos muestran que la totalidad de los controles positivos de óbex, presentaron el precipitado granular rojo indicativo de la inmunoreacción del anticuerpo frente a la $\mathrm{PrPsc}^{\mathrm{Sc}}$, lo que indica su localización en los núcleos nerviosos afectados, en cambio ninguno de los controles negativos de óbex presentó inmunotinción. Se utilizó el anticuerpo monoclonal F99/97.6.1, específico para detectar la $\mathrm{PrP}^{\mathrm{Sc}}$ por IHQ (O'Rourke y col 2000), además del cromógeno AEC para revelar la inmunoreacción (Spraker y col 2002, VMRD 2004). Algunas investigaciones utilizan la mezcla de dos anticuerpos monoclonales, para aumentar la detección de la $\operatorname{PrP}^{\mathrm{Sc}}$ a través de la secuencia conservada de la PrP, presente en las especies mamíferas que poseen EETs naturales (O'Rourke y col 2000, Spraker y col 2002). En este estudio que sólo utilizó un anticuerpo, no se encontraron diferencias en los controles positivos y negativos con los resultados descritos por O’Rourke y col (2002), quien utilizó los dos anticuerpos.

La observación microscópica de la totalidad de las muestras aptas para la IHQ, permitió determinar satisfactoriamente la presencia de tejido linfoide del tercer párpado, tanto en las muestras obtenidas en el país como en las aportadas por el CR. Además, fue posible evidenciar la inmunoreacción en los folículos linfoides de las muestras positivas y en los controles positivos de óbex, todas aportadas por el CR, así como también la ausen-
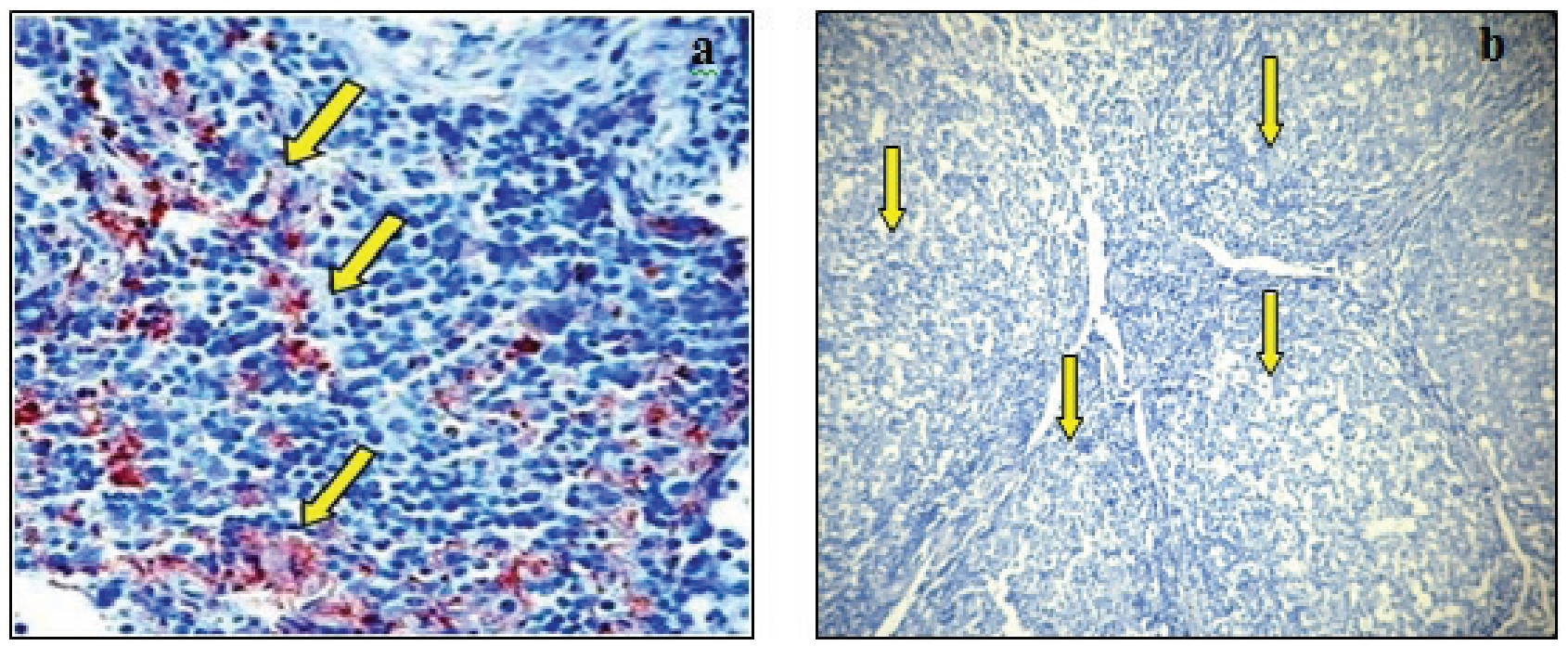

Figura 1. Imagen de tercer párpado. a) Muestra positiva, inmunoreacción del anticuerpo frente a la proteína priónica patológica, evidenciada por el inmunoprecipitado rojo en el centro germinal (flechas), (IHQ 400x). b) Muestra negativa, ausencia de inmunomarcaje en los centros germinales (flechas), (IHQ 200x).

Image of third eyelid. a) Positive sample, immunoreactivity of antibody against abnormal prion protein evidenced by the red immunoprecipitate in the germinal center (arrows) (IHC 400x). b) Negative sample, absence of immunoreactivity in the germinal centers (arrows) (IHC 200x). 

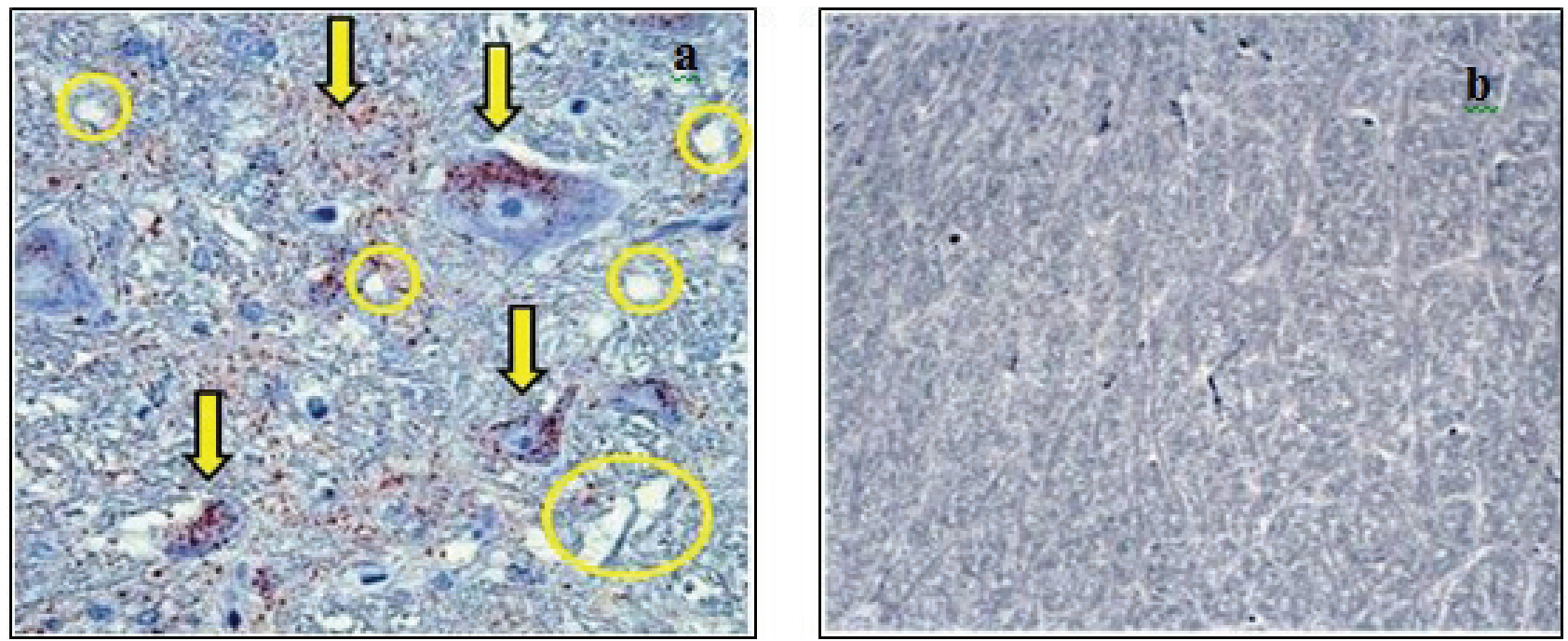

Figura 2. Imagen de óbex de ovinos. a) control positivo, se observa presencia de vacuolas (círculos) y el inmunoprecipitado rojo por la presencia de la PrPSc (flechas), (IHQ 200x), b) control negativo, no se observa presencia de inmunoprecipitado ni de vacuolas (IHQ 100x).

Image of obex of sheep. a) Positive control, vacuoles (circles) can be observed and the red immunostaining due to the presence of PrPSc (arrows), (IHC 200x), b) negative control, with no presence of vacuoles nor immunoprecipitated (IHC 100x).

cia de inmunomarcaje en las muestras nacionales y en los controles negativos. En el scrapie clásico, el desgaste crónico y la vECJ, la $\mathrm{PrP}^{\mathrm{Sc}}$ se acumula antes en el SLR y luego en el SNC, sin embargo, no existe asociación de lesiones patológicas en SLR, en contraste con el SNC donde las lesiones son bien conocidas (Monleón y col 2005, Aguzzi y col 2008, Vidal y col 2009).

El cuadro 2, resume los resultados obtenidos mediante la IHQ de la totalidad de las muestras aptas, incluyendo las aportadas y los controles del Centro de referencia. Así, del total de muestras de tercer párpado obtenidas para este estudio, las 74 clasificadas como aptas por H-E para la determinación de la $\mathrm{PrP}^{\mathrm{sc}}, 67(90,5 \%)$ resultaron negativas ( 61 obtenidas en el país y 6 del centro de referencia) y sólo 7 (9,5\%) aportadas por el centro de referencia fueron positivas a scrapie. En este estudio se analizó el tejido linfoide de tercer párpado de ovinos entre los 2 a 4 años, para aplicar la técnica independiente de la edad, se clasificaron las muestras según su aptitud, con lo cual se logró implementar y validar la técnica de IHQ para la detección del scrapie en muestras de tercer párpado ovino en las condiciones del laboratorio oficial de diagnóstico del país (SAG). Los resultados de este estudio indican que las muestras de tercer párpado son adecuadas para el diagnóstico de scrapie, sin embargo, su obtención in vivo presentaría como dificultad el inmovilizar manualmente al animal, por lo cual se podría invertir más tiempo.

Para que los métodos diagnósticos de priones sean realmente útiles, deben ser capaces de identificar los casos sospechosos en fases tempranas de la infección, característica que cumple el diagnóstico mediante tejido linfoide del tercer párpado, que permite identificar un animal positivo
Cuadro 2. Muestras aptas según su origen y resultado de IHQ. A suitable samples according to their origin and results of IHC.

\begin{tabular}{lccc}
\hline Origen de muestras & IHQ N $^{\circ}$ total & Positivas & Negativas \\
\hline Matadero TP & 24 & 0 & 24 \\
Con signos clínicos TP & 37 & 0 & 37 \\
Centro referencia TP & 13 & 7 & 6 \\
Centro referencia óbex & 13 & 7 & 6 \\
Control (+) óbex & 8 & 8 & 0 \\
Control (-) óbex & 8 & 0 & 8 \\
\hline Total & 103 & 22 & 81 \\
\hline
\end{tabular}

(TP: Tercer párpado).

a scrapie desde los 10 meses de edad, es decir mucho antes del comienzo de los signos clínicos (Vargas y col 2006, Grassi y col 2008). Las muestras de tercer párpado obtenidas en el país resultaron negativas a la detección de la $\mathrm{PrP}^{\mathrm{Sc}}$, puesto que no se observó presencia de inmunotinción en los folículos linfoides, la que sí se observó en algunas muestras de tercer párpado aportadas por el CR y en los controles positivos de óbex. Como en el tejido linfoide no es posible observar lesiones específicas indicativas de la presencia de priones, y a pesar que este hecho, remarca la eficacia de la detección inmunológica, sin embargo, presenta el inconveniente que puedan aumentar los falsos negativos, alcanzando diferentes porcentajes de sensibilidad (O'Rourke y col 2002, Monléon y col 2011). Esto se debe a la dinámica 
de replicación del prión, que puede estar influenciada por diferentes variables, una de ellas son las diferencias genotípicas del hospedador (Fediaevsky y col 2008, Ortiz-Pelaez y Bianchini 2011), sin embargo en rebaños de genética similar la diferencia más importante apuntaría al tipo de prión. Así, los falsos negativos en el estudio del SLR por IHQ no serían un problema de reconocimiento, sino de individualidades en la presentación de la enfermedad, como manifestación directa del tipo de cepa de $\mathrm{PrP}^{\mathrm{Sc}}$ involucrada (Benestard y col 2008, van Keulen y col 2008).

El diagnóstico de scrapie por IHQ utilizando muestras de tejido linfoide supone una importante herramienta para la vigilancia y el control, aunque como la sensibilidad no es $100 \%$ debe realizarse en conjunto con un programa de selección u otro método confirmatorio. Algunos países que poseen la enfermedad de manera endémica, tienen entre sus estrategias de control el monitoreo de animales vivos mediante la biopsia de tercer párpado (Detwiler y Baylis 2003). La instauración de esta estrategia de vigilancia permitiría al país contar con mayor información sanitaria del scrapie ovino.

En el presente trabajo se logró implementar y aplicar la técnica de IHQ en muestras de tejido linfoide de tercer párpado de ovinos, en el laboratorio de diagnóstico oficial para el país, la que podría aplicarse al diagnóstico de la enfermedad en la etapa preclínica o clínica de animales sospechosos (Vargas y col 2006, Vidal y col 2009), sin involucrar su sacrificio. Este estudio hace posible recomendar la implementación y aplicación de la IHQ en tejido linfoide, para un primer screening in vivo de los animales en la detección de scrapie, incorporada en los programas de vigilancia y control para la enfermedad.

\section{REFERENCIAS}

Aguzzi A, C Sigurdson, M Heikenwaelder. 2008. Molecular mechanisms of prion pathogenesis. Annu Rev Pathol Mech Dis 3, 11-40.

Benestad S, J Arsac, W Goldmann, M Nöremark. 2008. Atypical/Nor98 scrapie: properties of the agent, genetics, and epidemiology. Vet Res 39, 19-32.

Detwiler L, M Baylis. 2003. The epidemiology of Scrapie. Rev Sci Tech Off Int Epiz 22, 121-143.

Farías G, A Machuca, C Bravo, M Madariaga, C Jara, C Lecocq. 2009. Utilización de inmunoreacciones para detección de scrapie en óbex de ovinos provenientes de la XII Región de Chile. Av Cs Vet 24, 49-55.

Fediaevsky A, S Tongue, M Nöremark, D Calavas, G Ru, P Hopp. 2008. A descriptive study of the prevalence of atypical and classical scrapie in sheep in 20 European countries. BMC Vet Res 4, 1186-1210.

Gilch S, N Chitoor, Y Taguchi, M Stuart, J, Jewell, H Schatzl. 2011. Chronic wasting disease. Top Curr Chem 305, 51-77.

González L, M Dagleish, S Martin, G Dexter, P Steele, J Finlayson, M Jeffrey. 2008. Diagnosis of preclinical Scrapie in live sheep by the immunohistochemical examination of rectal biopsies. Vet Rec 162, 397-403.

Grassi J, S Maillet, S Simon, N Morel. 2008. Progress and limits of TSE diagnostic tools. Vet Res 39, 33-40.

Jeffrey M, L González. 2007. Classical sheep transmissible spongiform encephalopathies, pathogenesis, pathological phenotypes and clinical diseases. Neuropathol Appl Neurobiol 33, 373-394.

Monleón E, M Monzón, P Hortells, R Bolea, C Acín, F Vargas, J Badiola. 2005. Approaches to scrapie diagnosis by applying immunohistochemistry and rapid test on central nervous and lymphoreticular systems. J Virol Methods $125,165-171$.

Monleón E, M Garza, R Sarasa, J Álvarez, R Bolea, M Monzón, A Vargas, J Badiola, C Acin. 2011. An assessment of the efficiency of PrPsc detection in rectal mucosa and third-eyelid biopsies from animals infected with scrapie. Vet Microbiol 147, 237-243.

O'Rourke K, T Baszler, T Besser, J Miller, R Cutlip, G Wells, S Ryder, S Parish, A Hamir, N Cockett, A Jenny, D Knowles. 2000. Preclinical diagnosis of scrapie by immunohistochemestry of third eyelid lymphoid tissue. J Clin Microbiol 38, 3254-3259.

O'Rourke K, J Duncan, J Logan, A Anderson, D Norden, S Williams, B Combs, R Stobart, G Moss, D Sutton. 2002. Active surveillance for scrapie by third eyelid biopsy and genetic susceptibility testing of flocks of sheep in Wyoming. Clin Diag Lab Immunol 9, 966-971.

Ortiz-Pelaez A, J Bianchini. 2011. The impact of the genotype on the prevalence of classical scrapie at population level. Vet Res 42, 31-39.

Spraker T, K O'Rourke, A Balachandran, R Zink, B Cummings, W Miller, B Powers. 2002. Validation of monoclonal antibody F99/97.6.1 for immunohistochemical staining of brain and tonsil in mule deer (Odocoileus hemionus) with chronic wasting disease. J Vet Diagn Invest $14,3-7$.

Tranulis M, S Benestad, T Baron, H Kretzschmar. 2011. Atypical prion diseases in humans and animals. Top Curr Chem 305, 23-50.

van Keulen L, A Bossers, F van Zijderveld. 2008. TSE pathogenesis in cattle and sheep. Vet Res 39, 24-32.

Vargas F, L Luján, R Bolea, E, Monleón, I Martín-Burriel, A Fernández, I De Blas, J Badiola. 2006. Detection and clinical evolution of scrapie in sheep by $3^{\text {rd }}$ Eyelid biopsy. $J$ Vet Inter Med 20, 187-193.

Vidal E, C Acín, L Foradada, M Monzón, M Márquez, E Monleón, M Pumarola, J Badiola, R Bolea. 2009. Immunohistochemical characterisation of classical scrapie neuropathology in sheep. J Comp Path 141, 135-146.

VMRD Inc. 2004. Standard operating procedure for detection of prion protein $(\mathrm{PrP})$ in brain and lymphoid tissue of ruminant using monoclonal antibody immunohistochemistry. Version 4, USA.

Wadsworth J, I Dalmau-Mena, S Joiner, J Linehan, C O’Malley, C Powell, S Brandner, E Asante, J Ironside, D Hilton, J Collinge. 2011. Effect of fixation on brain and lymphoreticular vCJD prions and bioassay of key positive specimens from a retrospective vCJD prevalence study. $J$ Pathol 223, 511-518. 